\title{
EUD based 90Y-TOF-PET post selective internal radionuclide therapy provides a similar TCP in HCC than observed in EBRT
}

philippe D'ABADIE ( $\nabla$ philippe.dabadie@saintluc.uclouvain.be )

Cliniques universitaires Saint-Luc Institut Roi Albert II https://orcid.org/0000-0002-7725-2527

\section{Stephan Walrand}

Cliniques universitaires Saint-Luc Institut Roi Albert II

\section{Michel Hesse}

Cliniques universitaires Saint-Luc Institut Roi Albert II

\section{Ivan Borbath}

Cliniques universitaires Saint-Luc Institut Roi Albert II

\section{Renaud Lhommel}

Cliniques universitaires Saint-Luc Institut Roi Albert II

\section{François Jamar}

Cliniques universitaires Saint-Luc Institut Roi Albert II

\section{Research Article}

Keywords: HCC, TCP, EUD, PET, FDG, acetate

Posted Date: March 7th, 2022

DOI: https://doi.org/10.21203/rs.3.rs-1388667/v1

License: (1) This work is licensed under a Creative Commons Attribution 4.0 International License. Read Full License 


\section{Abstract}

Background: Tumor equivalent uniform dose (EUD) is a predictor of patient outcome after selective internal radionuclide therapy (SIRT) of hepatocellular carcinoma (HCC) and can be evaluated with ${ }^{90} \mathrm{Y}$ TOF-PET. The aim is to evaluate the correlation between PET based tumors EUD and the clinical response evaluated with dual molecular tracer $\left({ }^{11} \mathrm{C}\right.$-acetate and ${ }^{18} \mathrm{~F}$-FDG) PET/CT post SIRT.

Methods: 34 HCC tumors in 22 patients were prospectively evaluated. The metabolic response was characterized by the Total Lesion Metabolism variation ( $\triangle T L M)$ between baseline and follow up. This response allowed to compute a Tumor Control Probability (TCP) as a function of the tumor EUD.

Results: The dose response correlation was exponential-asymptotic shaped and highly significant $(R=0.72, P<0.001)$. With a cutoff of $40 G y$, the metabolic response was strongly different in both groups (median response $35 \%$ versus $100 \%, p<0.001$ ). Moreover, the TCP (EUD) was very similar to that observed in External Beam Radiation Therapy (EBRT). Conclusions: EUD based ${ }^{90} \mathrm{Y}$ TOF-PET/CT predicts the metabolic response after SIRT of HCC assessed using dual molecular PET tracers and provides a similar TCP curve to that observed in EBRT. Both TCPs show that a EUD of 100 Gy is needed and sufficient to control a HCC.

\section{Background}

Liver selective internal radionuclide therapy (SIRT) is part of the treatment strategy of hepatocellular carcinoma (HCC) [1] and aims to deliver high efficient doses to tumors [2]. The tumor-absorbed dose is a predictive factor of the treatment's effectiveness [3]. Comparing the different types of radiation therapies, the absorbed dose efficacy cutoff for glass microspheres is twice that for resin microspheres and up to five times more than with external beam radiation therapy (EBRT)[4-7]. The heterogeneous dose distribution in SIRT explains these differences and can be accurately revealed with Time of Flight (TOF)$P E T{ }^{90} \mathrm{Y}$ imaging $[8,9]$ when taking into account the Equivalent Uniform dose (EUD)[10]. EUD reunifies the efficacy threshold doses in SIRT (performed with resin or glass microspheres) but also in EBRT [11]. Previous radiobiological models developed in SIRT for HCC demonstrated an exponential correlation between the tumor-absorbed dose and the clinical response assessed with conventional imaging $[5,12$, 13]. In colorectal liver metastases, a similar correlation was demonstrated using the metabolic response assessed with ${ }^{18} \mathrm{~F}$-FDG PET/CT $[14,15]$.

Functional imaging (PET/CT) is currently not recommended for the management of HCC, especially because ${ }^{18} \mathrm{~F}$-FDG PET/CT has a low sensitivity, with a definite uptake in less than $50-65 \%$ of the cases[16]. ${ }^{11} \mathrm{C}$-Acetate PET/CT is more sensitive for HCC detection, with an uptake reaching 85\%[17].

${ }^{11} \mathrm{C}$ acetate enters the Krebs cycle as a substrate for $\beta$-oxidation in fatty acid and cholesterol synthesis which is likely the explanation of its uptake by differentiated HCC [17]. On the other hand, the high level of glucose-6-phosphatase in differentiated HCC leads to the release of ${ }^{18} \mathrm{FDG}$ while poorly differentiated 
HCC, which has a low abundance of this enzyme, tends accumulate ${ }^{18} \mathrm{~F}-\mathrm{FDG}[16,17]$. As a result, performing a dual molecular tracer PET/CT acquisition $\left({ }^{11} \mathrm{C}\right.$ - acetate and $\left.{ }^{18} \mathrm{~F}-\mathrm{FDG}\right)$ was able to improve this sensibility up to $98 \%[18]$.

This study aims to demonstrate a radiobiological model in HCC built on ${ }^{90} \mathrm{Y}$ TOF- PET/CT based EUD and the metabolic response assessed with dual molecular PET tracer.

\section{Methods}

Patients

$23 \mathrm{HCC}$ patients, referred to our department for SIRT, were prospectively enrolled in this study after their informed consent and approval by the local ethics committee (2015/010CT/522).

Each patient was evaluated with dual isotope $\left({ }^{11} \mathrm{C}\right.$-acetate and $\left.{ }^{18} \mathrm{~F}-\mathrm{FDG}\right) \mathrm{PET} / \mathrm{CT}$ at baseline and at follow-up (FU) 2 and 4 months after SIRT. 4 patients received a second treatment after an interval of 5 months and were evaluated again. 4 patients had multifocal tumors (2 to 6 ) while the majority had only one tumor.

A total of 34 tumors demonstrated a metabolic uptake, $80 \%$ were positive with acetate PET/CT, $52 \%$ with FDG PET/CT and $40 \%$ with both imaging. In one patient, the tumor uptake was not significant with either PET tracers and the metabolic evaluation was not possible, resulting in 22 studied patients. No other exclusion factor was applied.

The patients characteristics are summarized in Table 1.

Table 1

Patients characteristics

\begin{tabular}{|ll|}
\hline Characteristics & Data \\
\hline Age (Years) & $73(68-79)$ \\
\hline Sex & Male: 14 (64\%) \\
\cline { 2 - 2 } & Female: 8 (34\%) \\
\hline BCLC staging & A: $8(36 \%)$ \\
\cline { 2 - 2 } & B: $8(36 \%)$ \\
\hline C: $6(28 \%)$ \\
\hline Tumor volume $(\mathrm{ml})$ & $36(11-97)$ \\
\hline
\end{tabular}

Continuous variables described with median and 95\% confidence interval displayed between parentheses. BCLC: Barcelona Clinic Liver Cancer 
Treatment

SIRT was performed according to standard recommendations [19]. Resin microspheres (Sir-Spheres ${ }^{\circledR}$, Sirtex Medical Ltd., Sydney, Australia) and glass microspheres (Therasphere ${ }^{\circledR}$, Boston Scientific, Boston, MA) were used in 17 and 9 treatments, respectively.

A 40-min 2-bed positions ${ }^{90} \mathrm{Y}$ TOF-PET scan (Gemini TF64 Philips Medical Systems, Cleveland, OH) was acquired within $4 \mathrm{~h}$ following the SIRT. Reconstruction was performed with the 3D line of response (LOR)-TOF blob-based OSEM algorithm from Philips with 2 iterations times 33 subsets and a 4x4 mm3 voxel size. ${ }^{90} \mathrm{Y}$ activity distribution was transformed into a 3D-map of absorbed doses using a previously validated method [20]. In summary, voxel counts were converted to absorbed dose distribution by convolving the ${ }^{90} \mathrm{Y}$ activity distribution with a dose point kernel, after spatial resolution recovery.

\section{Tumor response assessment}

Whole body TOF-PET/CT acquisition (Gemini TF 64, Philips Medical Systems, Cleveland, OH) was acquired 20 minutes after intravenous injection of ${ }^{11} \mathrm{C}$-acetate (370-540 MBq, half-life: 20 minutes). 30 minutes after this first acquisition, ${ }^{18} \mathrm{~F}-\mathrm{FDG}$ was injected (280-310 MBq) and images were acquired after an incorporation of 60 minutes. Acquisitions were performed at baseline and during FU at 2 and 4 months after treatment.

Tumor contours were automatically defined with acetate or FDG PET/CT using an isocontour method (41\% SUVmax threshold) performed with the MIM software (V7.1, MIM Software Inc., Cleveland, $\mathrm{OH}$ ).

For each patient, the tracer, i.e., ${ }^{11} \mathrm{C}$-acetate or ${ }^{18} \mathrm{~F}-\mathrm{FDG}$, giving the highest normal liver to tumor tissue ratio on the baseline PET imaging was selected to assess the tumor response metabolism.

For each tumor and each time point, the total lesion metabolism (TLM) was defined as:

$$
\mathrm{TLM}=\mathrm{SUV}_{\text {mean }} \times \text { Volume }
$$

1

The $\triangle T L M$ between baseline $(B)$ and FU defined the metabolic response:

$\Delta \operatorname{TLM}(\backslash \%)=\frac{\mathrm{TLM}_{\mathrm{B}}-\mathrm{TLM}_{\mathrm{FU}}}{\mathrm{TLM}_{\mathrm{B}}} .100(2)$

The best metabolic response between the baseline and either 2- or 4-month was considered (corresponding to the maximal effects of radiations).

No TLM cutoffs are established to classify the metabolic response of tumors. However, regarding EUD, a threshold of 40 Gy was previously defined as an efficacy cutoff in HCC [11] and corresponded also to the efficacy cutoff in EBRT [7]. Therefore, this cutoff was applied for comparing the metabolic responses. 
Tumor dosimetry

Tumor contours were matched with the 3D map of absorbed doses, as previously defined, using a rigid co-registration with the MIM software.

Equivalent uniform dose (EUD) was calculated according to the Jones and Hoban formalism [21]:

$$
\mathrm{EUD}=-\frac{1}{\alpha} \ln \left(\frac{\sum_{\mathrm{i}} \mathrm{e}^{-\alpha \mathrm{D}_{\mathrm{i}}}}{\mathrm{N}}\right)
$$

3

where $\mathrm{a}$ is the apparent HCC radiosensitivity, $\mathrm{N}$ the number of voxels in the tumor and Di the absorbed dose in tumor voxel i. The terminology apparent radiosensitivity was introduced by Chiesa et al. [13] in order to take into account that this value depends on the spatial resolution of the imaging system as shown in [22]. In a previous ${ }^{90} \mathrm{Y}$ TOF-PET based EUD analyses, the apparent a coefficient was estimated to be $0.038 \mathrm{~Gy}^{-1}$ [11], i.e., about tenfold lower than the intrinsic in vitro $\mathrm{HCC} 0.40 \mathrm{~Gy}^{-1}$ radiosensitivity [23].

The Tumor Control Probability (TCP) was determined in consecutive EUD bins of $20 \mathrm{~Gy}$. Tumor control was defined as a metabolic response with $\triangle T L M \geq 0.80$, the value giving the best sigmoid shaped curve.

\section{Statistical analysis}

Wilcoxon signed rank test was used to compare groups. The correlation was analyzed using a Spearman coefficient (R).

Analyses were conducted with Prism software (version 7.0, GraphPad Software, La Jolla, Ca).

\section{Results}

The metabolic response correlated well with EUD (Fig. 1): the larger the EUD the higher the metabolic response. This dose response correlation was linear-exponential and highly significant according to the Spearman coefficient $(R=0.72, P<0.001)$. Moreover, the metabolic response to radiations was very similar for tumors positive with FDG PET/CT and with acetate PET/CT (Fig. 1). The metabolic response was significantly higher in tumors receiving more than $40 \mathrm{~Gy}$ (median $\triangle \mathrm{TLM}: 1.0$ ) compared to tumors receiving a lower dose (median $\triangle$ TLM: $0.35, \mathrm{P}<0.001$ ).

\section{Discussion}

This study confirms the strong correlation already observed between reduction of tumor metabolism and EUD $[13,14]$, as well as between the TCP and the tumor EUD $[12,13]$. A novelty of the present observation is that this correlation holds true when mixing acetate and FDG data for the tumor metabolism 
assessment. This makes sense considering the fact that reduction of acetate or FDG uptake mainly results from the reduction of tumor living cells number. Indeed, proteins which constitute the metabolic engines are hugely radioresistant. For instance, a dose of $100 \mathrm{~Gy}$ damages less than $5 \%$ of albumin (Figure. 1a in [25]) whereas $40 \mathrm{~Gy}$, assuming a radiosensitivity $\mathrm{a}=0.4 \mathrm{~Gy}^{-1}$ [23], kills $1-\mathrm{e}^{-0.4 \times 40} \approx$ $99.99999 \%$ of HCC cells [23]. Finally, the use of ${ }^{90} \mathrm{Y}$ TOF-PET to compute the dose, rather than MAA or bremsstrahlung SPECT used in previous studies [12-14], improved the correlation.

Beyond this dose-response confirmation, the main finding of the study is that the observed TCP as a function of the EUD in SIRT is very similar to that of EBRT. We already demonstrated [26] that ${ }^{90} \mathrm{Y}$ TOFPET based EUD provided the same threshold to discriminate "responding" and "non- responding" patients in glass SIRT, resin SIRT and EBRT, i.e., 40Gy. These results proved that ${ }^{90} \mathrm{Y}$ TOF-PET based EUD rightly takes into account the impact of the heterogeneity of the dose distribution. In addition, the similarity of the SIRT and EBRT TCPs consolidates that to surely control a tumor, i.e., TCP $\approx 1$, the needed and sufficient EUD is $100 \mathrm{~Gy}$. The sigmoid shape of the TCP curve enlightens the importance to reach at least a EUD of $100 \mathrm{~Gy}$ in each tumor and emphasize the necessity to optimize the treatment planning liver to reach this goal $[3,27,28]$. Note that the absorbed dose $D$ needed to achieve this EUD can vary from 190 to 1800 Gy depending on the dose distribution heterogeneity (supplementary material, appendix A).

The observed SIRT TCP also explains why the EUD $=40$ Gy threshold well split the patient survival curves into 2 clear different groups [26]. Indeed, this threshold correspond to the EUD50, as a result one group contains a majority of responding patients and the other one a majority of non-responding patients.

The optimal $\triangle T L M 80 \%$ threshold, and not $100 \%$, used to obtain a well-shaped TCP sigmoid curve results from different effects: errors in the TLM assessment, FU delay too short to allow all the tumor cells to die or in contrary FU delay sufficiently long enough to allow a repopulation of the tumor site by healthy liver tissue which also takes up ${ }^{11} \mathrm{C}$-acetate or ${ }^{18} \mathrm{~F}$-FDG.

This study has some limitations. First, analyses were performed in a limited number of patients and tumors compared to previous reported studies [12-14]. However, the better accuracy of ${ }^{90} \mathrm{Y}$ TOF-PET in dose distribution assessment, versus MAA or bremsstrahlung SPECT, reduces the statistical blurring, allowing to get a good correlation with a reduced number of patients. Only few tumors received low absorbed doses and demonstrated a poor metabolic response, limiting the accuracy of our radiobiological model for low doses. Second, the follow up was 4 months at maximum but some tumors could respond later to radiations and hence the effects of radiations could be sometimes underestimated, justifying the choice of the threshold $\triangle T L M=80 \%$ rather than $100 \%$ in the TCP derivation [2].EUD is based on a single alpha value and it is likely that the radiosensitivity may vary between HCC occurring within different clinical entities. Accordingly, this delayed response may explain why some tumors did not disclose complete metabolic response 4 months after therapy despite a very efficient EUD.

\section{Conclusions}


${ }^{90} \mathrm{Y}$-TOF-PET based EUD is strongly correlated with the metabolic response in HCC assessed by dual molecular tracer PET imaging and is associated with a TCP close to that in EBRT. ${ }^{90} \mathrm{Y}$-TOF-PET based EUD reconciles dose levels in SIRT and EBRT. The use of this parameter must be encouraged in future dosimetry studies for a better understanding of the toxicity and efficacy threshold doses in SIRT.

\section{Abbreviations}

EBRT: External beam radiation therapy

EUD: Equivalent Uniform dose

FU: Follow-up

HCC: Hepatocellular carcinoma

RE: Radioembolization

TCP: Tumor control probability

TLM: Total lesion metabolism

\section{Declarations}

\section{Ethics approval and conent to participate}

The study was conducted in accordance with the Declaration of Helsinki, and approved by the Ethics Committee of UniversitéCatholique de Louvain (2015/010CT/522). Informed consent was obtained from all subjects involved in the study.

\section{Consent for publication}

Not applicable

\section{Availability of data and materials}

Data generated in the study can be found at the Mendeley repository website at: https://doi.org/10.17632/x68ys6nz5d.1

\section{Competing interests}

The Authors, PdA, SW, MH, IB, RL and FJ, declare no financial or non-financial competing interests.

\section{Funding}

Authors declare no funding source. 


\section{Authors' contributions}

Conceptualization, PdA, SW and FJ.; methodology, PdA, SW and MH .; software, $\mathrm{MH}$; validation, $\mathrm{PdA}$, SW. and FJ.; formal analysis, PdA and FJ.; investigation, IB and RL.; resources, RL, IB; data curation, PdA.; writing-original draft preparation, PdA.; writing-review and editing, SW and FJ.; visualization, FJ.; supervision, FJ.; project administration, FJ.; funding acquisition, none. All authors have read and agreed to the final manuscript.

\section{Acknowledgements}

Not applicable

\section{References}

1. Marrero JA, Kulik LM, Sirlin CB, Zhu AX, Finn RS, Abecassis MM, Roberts LR. and J. K. Heimbach. Diagnosis, staging, and management of hepatocellular carcinoma: 2018 practice guidance by the american association for the study of liver diseases. Hepatology. 2018;68:723-50.

2. Sangro B, Inarrairaegui M. and J. I. Bilbao. Radioembolization for hepatocellular carcinoma. J Hepatol. 2012;56:464-73.

3. Garin E, Tselikas L, Guiu B, Chalaye J, Edeline J, de Baere T, Assenat E, Tacher V, Robert C, TerroirCassou-Mounat $\mathrm{M}$, et al. Personalised versus standard dosimetry approach of selective internal radiation therapy in patients with locally advanced hepatocellular carcinoma (dosisphere-01): A randomised, multicentre, open-label phase 2 trial. Lancet Gastroenterol Hepatol. 2021;6:17-29.

4. Garin E, Lenoir L, Edeline J, Laffont S, Mesbah H, Poree P, Sulpice L, Boudjema K, Mesbah M, Guillygomarc'h A, et al. Boosted selective internal radiation therapy with 90y-loaded glass microspheres (b-sirt) for hepatocellular carcinoma patients: A new personalized promising concept. Eur J Nucl Med Mol Imaging. 2013;40:1057-68.

5. Hermann AL, Dieudonne A, Ronot M, Sanchez M, Pereira H, Chatellier G, Garin E, Castera L, Lebtahi R, Vilgrain $\mathrm{V}$, et al. Relationship of tumor radiation-absorbed dose to survival and response in hepatocellular carcinoma treated with transarterial radioembolization with (90)y in the sarah study. Radiology. 2020;296:673-84.

6. d'Abadie P, Walrand S, Hesse M, Annet L, Borbath I, Van den Eynde M, Lhommel R, Jamar F. Prediction of tumor response and patient outcome after radioembolization of hepatocellular carcinoma using 90y-pet-computed tomography dosimetry. Nucl Med Commun. 2021;42:747-54.

7. Seong J, Park HC, Han KH, Chon CY, Chu SS, Kim GE. and C. O. Suh. Clinical results of 3-dimensional conformal radiotherapy combined with transarterial chemoembolization for hepatocellular carcinoma in the cirrhotic patients. Hepatol Res. 2003;27:30-5.

8. Pasciak AS, Bourgeois AC, McKinney JM, Chang TT, Osborne DR, Acuff SN. and Y. C. Bradley. Radioembolization and the dynamic role of (90)y pet/ct. Front Oncol. 2014;4:38. 
9. Walrand S, Hesse M, Chiesa C, Lhommel R, Jamar F. The low hepatic toxicity per gray of 90y glass microspheres is linked to their transport in the arterial tree favoring a nonuniform trapping as observed in posttherapy pet imaging. J Nucl Med. 2014;55:135-40.

10. Hesse M, d'Abadie P, Lhommel R, Jamar F, Walrand S. Yttrium-90 tof-pet-based eud predicts response post liver radioembolizations using recommended manufacturer fdg reconstruction parameters. Front Oncol. 2021;11:592529.

11. d'Abadie P, Hesse M, Jamar F, Lhommel R. and S. Walrand. (90)y tof-pet based eud reunifies patient survival prediction in resin and glass microspheres radioembolization of hcc tumours. Phys Med Biol. 2018;63:245010.

12. Strigari L, Sciuto R, Rea S, Carpanese L, Pizzi G, Soriani A, laccarino G, Benassi M, Ettorre GM. and C. L. Maini. Efficacy and toxicity related to treatment of hepatocellular carcinoma with 90y-sir spheres: Radiobiologic considerations. J Nucl Med. 2010;51:1377-85.

13. Chiesa C, Mira M, Maccauro M, Spreafico C, Romito R, Morosi C, Camerini T, Carrara M, Pellizzari S, Negri A, et al. Radioembolization of hepatocarcinoma with (90)y glass microspheres: Development of an individualized treatment planning strategy based on dosimetry and radiobiology. Eur $\mathrm{J} \mathrm{Nucl}$ Med Mol Imaging. 2015;42:1718-38.

14. Flamen P, Vanderlinden B, Delatte P, Ghanem G, Ameye L, Van Den Eynde M, Hendlisz A. Multimodality imaging can predict the metabolic response of unresectable colorectal liver metastases to radioembolization therapy with yttrium-90 labeled resin microspheres. Phys Med Biol. 2008;53:6591-603.

15. Levillain H, Duran Derijckere I, Marin G, Guiot T, Vouche M, Reynaert N, Hendlisz A, Vanderlinden B, Flamen P. (90)y-pet/ct-based dosimetry after selective internal radiation therapy predicts outcome in patients with liver metastases from colorectal cancer. EJNMMI Res. 2018;8:60.

16. Sacks A, Peller PJ, Surasi DS, Chatburn L, Mercier G. and R. M. Subramaniam. Value of pet/ct in the management of primary hepatobiliary tumors, part 2. AJR Am J Roentgenol. 2011;197:W260-5.

17. Park JW, Kim JH, Kim SK, Kang KW, Park KW, Choi JI, Lee WJ, Kim CM. and B. H. Nam. A prospective evaluation of $18 \mathrm{f}-\mathrm{fdg}$ and $11 \mathrm{c}$-acetate pet/ct for detection of primary and metastatic hepatocellular carcinoma. J Nucl Med. 2008;49:1912-21.

18. Ho CL, Chen S, Yeung DW, Cheng TK. Dual-tracer pet/ct imaging in evaluation of metastatic hepatocellular carcinoma. J Nucl Med. 2007;48:902-9.

19. Padia SA, Lewandowski RJ, Johnson GE, Sze DY, Ward TJ, Gaba RC, Baerlocher MO, Gates VL, Riaz A, Brown DB, et al. Radioembolization of hepatic malignancies: Background, quality improvement guidelines, and future directions. J Vasc Interv Radiol. 2017;28:1-15.

20. Lhommel R, van Elmbt L, Goffette P, Van den Eynde M, Jamar F, Pauwels S, Walrand S. Feasibility of $90 \mathrm{y}$ tof pet-based dosimetry in liver metastasis therapy using sir-spheres. Eur $\mathrm{J}$ Nucl Med Mol Imaging. 2010;37:1654-62.

21. Jones LC, Hoban PW. Treatment plan comparison using equivalent uniform biologically effective dose (eubed). Phys Med Biol. 2000;45:159-70. 
22. Hesse M, d'Abadie P, Lhommel R, Jamar F, Walrand S. Yttrium-90 tof-pet-based eud predicts response post liver radioembolizations using recommended manufacturer fdg reconstruction parameters. Front Oncol. 2021;11:3876.

23. Wigg AJ, Palumbo K, Wigg DR. Radiotherapy for hepatocellular carcinoma: Systematic review of radiobiology and modeling projections indicate reconsideration of its use. J Gastroenterol Hepatol. 2010;25:664-71.

24. Lausch A, Sinclair K, Lock M, Fisher B, Jensen N, Gaede S, Chen J, Wong E. Determination and comparison of radiotherapy dose responses for hepatocellular carcinoma and metastatic colorectal liver tumours. Br J Radiol. 2013;86:20130147.

25. Mishra K, Ojha H, Kallepalli S, Alok A, Chaudhury NK. Protective effect of ferulic acid on ionizing radiation induced damage in bovine serum albumin. Int J Radiation Res. 2014;12:113.

26. d'Abadie P, Hesse M, Jamar F, Lhommel R. and S. Walrand. 90y tof-pet based eud reunifies patient survival prediction in resin and glass microspheres radioembolization of hcc tumours. Phys Med Biol. 2018;63:245010.

27. Chiesa C, Mira M, Bhoori S, Bormolini G, Maccauro M, Spreafico C, Cascella T, Cavallo A, De Nile MC, Mazzaglia S, et al. Radioembolization of hepatocarcinoma with (90)y glass microspheres: Treatment optimization using the dose-toxicity relationship. Eur J Nucl Med Mol Imaging. 2020;47:3018-32.

28. d'Abadie P, Walrand S, Hesse M, Amini N, Lhommel R, Sawadogo K, Jamar F. Accurate non-tumoral 99mtc-maa absorbed dose prediction to plan optimized activities in liver radioembolization using resin microspheres. Phys Med. 2021;89:250-57.

\section{Figures}




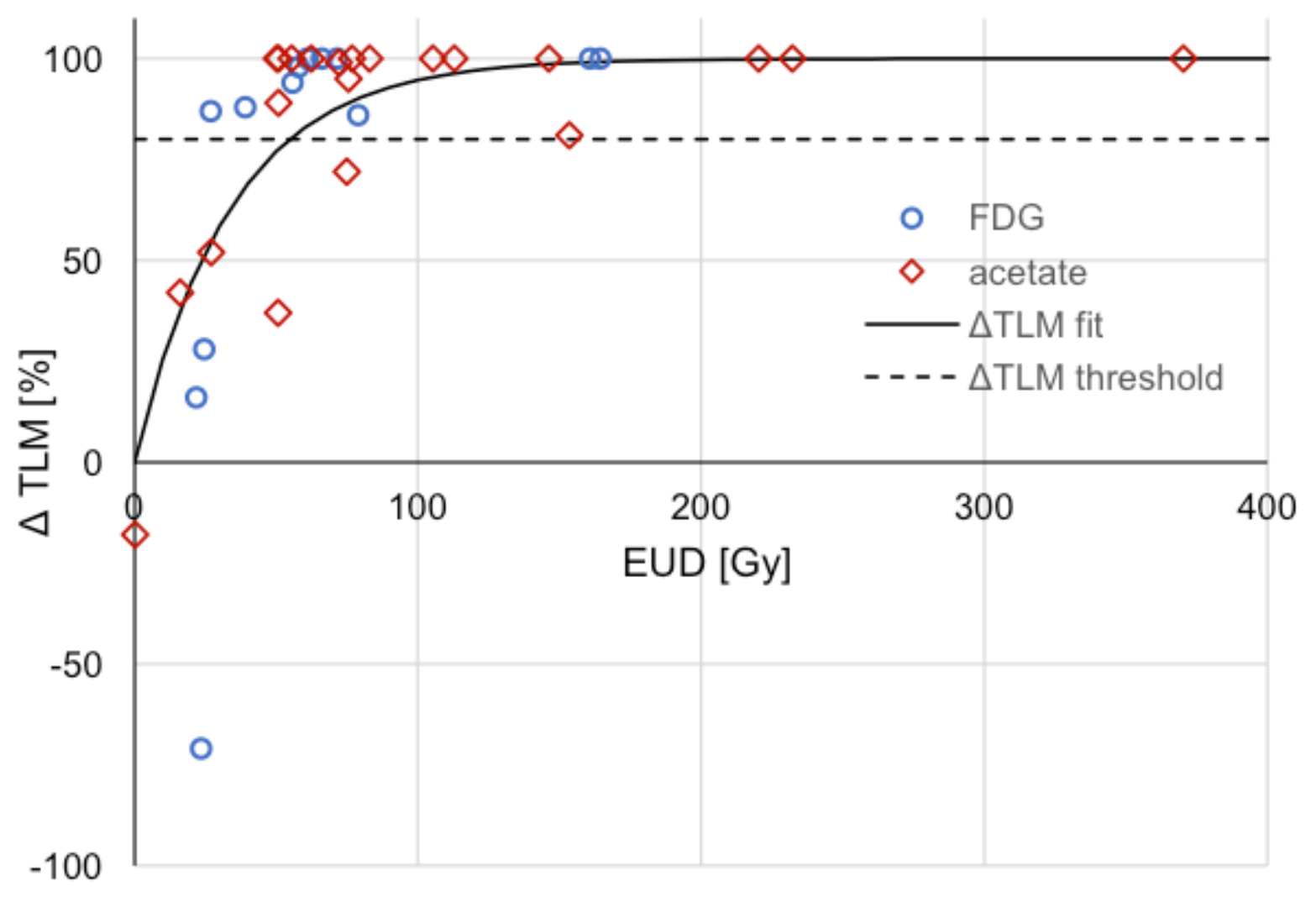

Figure 1

$\triangle T L M$ as a function of the EUD obtained from post-SIRT ${ }^{90} \mathrm{Y}$ TOF-PET. Blue circles: measured on ${ }^{11} \mathrm{C}$ acetate PET. Red diamonds: measured on ${ }^{18} \mathrm{~F}$-FDG PET. Dashed line: EUD threshold used to discriminate responding and not responding tumor in the TCP computation. 


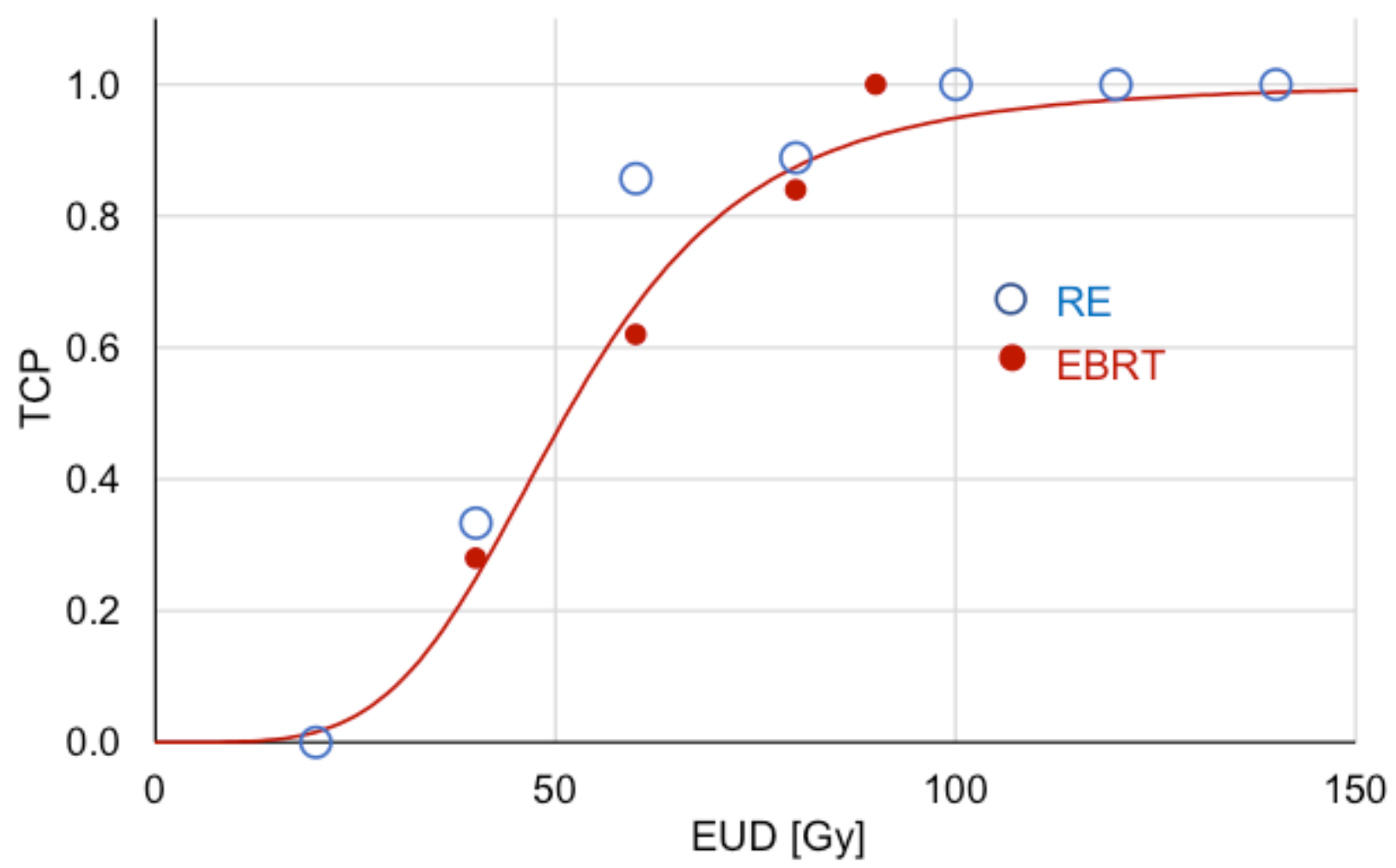

Figure 2

TCP as a function of the EUD. Blues circles: this SIRT study. Red bullets: External Beam Radiation Therapy (EBRT) derived from Lausch et al.[24]. Red solid line: EBRT TCP fitted with a logistic function.

\section{Supplementary Files}

This is a list of supplementary files associated with this preprint. Click to download.

- Supplementarymaterial.docx 